\title{
473972 - SIMULATORS CAN SHORTEN THE TIME TO PERFORM KEY THERAPEUTIC MANEUVERS
}

\author{
Rob Johnston, MD, Sanjiv Gupta, MD, Robert Elliott, MD, George Dumitrascu, \\ MD, Michelle Chiu, MD, Simone Crooks, MD \\ Anesthesiology, University of Ottawa, Ottawa, ON, Canada
}

Introduction: Scant evidence exists to show that high-fidelity simulations improve clinical performance(1). One reason for this paucity is the lack of a widely used validated performance measurement tool. We sought to determine whether repeated medical simulations improve performance among trainees as measured by objective, clinically relevant parameters.

Methods: With Ethics Board approval, 17 simulator-naïve junior Anesthesiology resident volunteers underwent a medical simulation during which a patient develops unstable atrial fibrillation. Primary outcome measure was time to cardioversion. Secondary outcomes were times to initiate other critical actions (give oxygen, apply monitors, obtain IV access, get help). Three months after the first simulation, participants took part in a second simulated scenario in which they were again presented with unstable Afib, but this time in a completely different clinical setting.

Results: 16 of 17 residents cardioverted the simulated patient in both scenarios (fig. 1). 1 resident did not perform cardioversion in the first simulation. There was a significant decrease in the time to cardioversion between the first and second simulation (15.0 v. $10.3 \mathrm{~min}, \mathrm{p}=0.0016,1$-sided paired t-test). There was a significant decrease in the time before residents called for "help" during the simulations (6.4 v. $3.1 \mathrm{~min}, \mathrm{p}=0.003)$. No other secondary outcome was significantly different. There was no increment between the sessions in the self-reported total number of cardiac arrests attended by the trainees (5.1 v. 5.4, $\mathrm{p}=\mathrm{NS}$ ).

Discussion: We showed that repeating a simulation led to a significant and clinically important decrease of almost 5 minutes in the time required to perform cardioversion on a simulated patient with unstable atrial fibrillation. We believe that the time to performing a key therapeutic intervention such as cardioversion is an objective, clinically relevant, and implicitly valid measure of decision-making ability. The lack of increase in the number of cardiac arrests attended by the participants between the sessions suggests that the improved performance is due to an effect of the simulation, rather than the additional three months of training. Our findings support the use of simulators to provide trainees with an opportunity to rehearse scripted actions that would be required in an emergency. References: 1. Can J Anesth 2004 51:5 455-464 


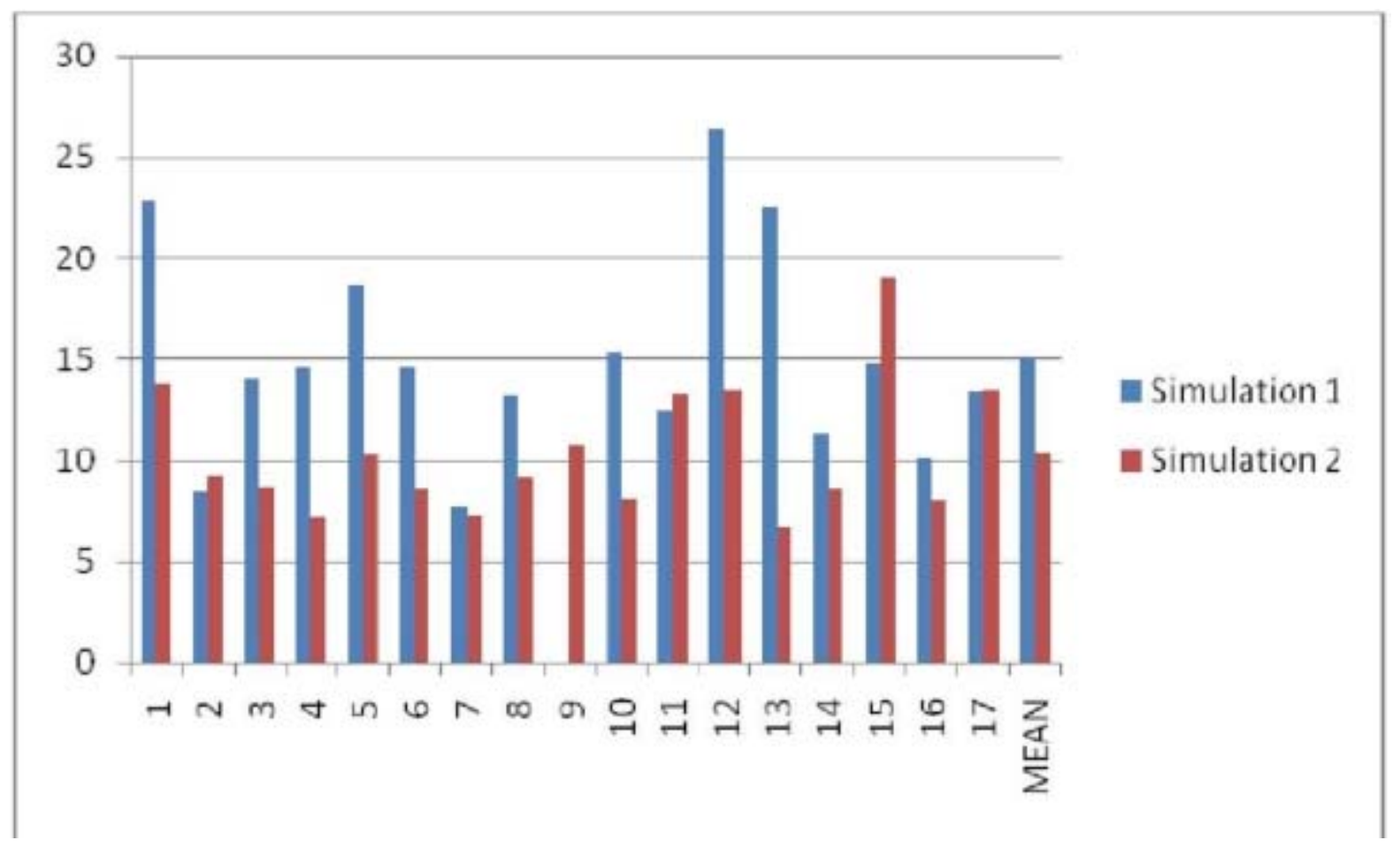

Fig. 1 Time to Perform Electrical Cardioversion of a Simulated Patient with Unstable Atrial Fibrillation. Vertical axis = time in minutes until cardioversion. Horizontal axis = individual resident. Light/blue bars = first simulation session; Heavy/red bars = second simulation 3 months later. Note that Resident \#9 did not cardiovert at the first session 